\title{
An elderly man presenting with an acute upper gastrointestinal bleed: a case report
}

\author{
Riaz A Agha
}

Address: Department of Gastroenterology, Hinchingbrooke Health Care NHS Trust, Hinchingbrooke Hospital, Hinchingbrooke Park, Huntingdon, Cambridgeshire, PE29 6NT, UK

Email:mail@riazagha.com

Received: 25 May 2009 Accepted: 19 June 2009 Published: 12 August 2009

Cases Journal 2009, 2:795। doi: |0.4076/|757-|626-2-795।

This article is available from: http://casesjournal.com/casesjournal/article/view/795 I

(c) 2009 Agha; licensee Cases Network Ltd.

This is an Open Access article distributed under the terms of the Creative Commons Attribution License (http://creativecommons.org/licenses/by/3.0), which permits unrestricted use, distribution, and reproduction in any medium, provided the original work is properly cited.

\begin{abstract}
An 80 year old man presented to the Accident and Emergency Department complaining of "black stools", increasing shortness of breath, chest tightness and epigastric pain. An upper gastro-intestinal bleed was diagnosed and the patient was managed conservatively with aggressive resuscitation and close monitoring. An oesophogastroduodenoscopy found no cause for the bleeding which ceased and the patient was discharged with a general practitioner follow-up.
\end{abstract}

\section{Introduction}

Acute upper gastrointestinal haemorrhage (AUGH) is a common medical emergency with a significant associated mortality [1] despite ongoing advances in its management [2].

Following decades of research, the introduction of risk stratification and prognostic scoring in the Rockall Score has allowed for the early division of this pool of patients into low or high risk of re-bleeding or death. This allows for the more effective management of resources and has brought greater awareness to the need for close monitoring of high risk patients who can decompensate suddenly on busy wards and admissions units.

\section{Case presentation}

A retired 80 year old Caucasian man presented to the Accident and Emergency Department complaining of "black stools", increasing shortness of breath, chest tightness and epigastric pain. Three days previously he had celebrated his birthday when he noted epigastric pain. This was similar in nature to the pain from his duodenal ulcers 20 years ago (managed conservatively at the time). The pain was relieved by eating and settled with simple analgesia and rest. From that point, the patient had black stools daily with decreasing exercise tolerance and shortness of breath on exertion. His past medical history included atrial fibrillation (AF), hypertension, and ischaemic heart disease with a bare metal stent inserted 6 weeks prior to admission in the left anterior descending branch. The patient was taking aspirin and warfarin for AF. The patient was a non-smoker, with minimal alcohol intake, a body mass index (BMI) of 25 and with no family history of note.

The patient was found to be normotensive at 130/80, but with a postural diastolic drop of $15 \mathrm{mmHg}$ with a pulse of 80 , decreased skin turgor, oxygen saturation of $98 \%$ on air and a respiratory rate of 16 . Routine blood tests found that Haemoglobin $(\mathrm{Hb})$ was $12.5 \mathrm{~g} / \mathrm{dL}$, with a urea/creatinine 
ratio $>100$ and an International Normalised Ratio (INR) of 2.3. On examination the patient was found to have mild tenderness in the epigastric region. The patient's initial Rockall score was thus four which carries a $24.6 \%$ risk of mortality [3].

The patient was treated with normal saline IV fluids and his anticoagulants were stopped. Reversal of warfarin with vitamin $\mathrm{K}$ was not deemed necessary. Fluid and stool charts together with regular observations were initiated. Oesophogastroduodenoscopy (OGD) was scheduled to take place within 72 hours of admission. During the period prior to OGD, the patient's blood pressure dropped to $110 / 70$, pulse increased to 95 and $\mathrm{Hb}$ decreased to $10.3 \mathrm{~g} / \mathrm{dL}$. The patient did not become symptomatic and a blood transfusion was not performed although a group had already been taken. Fluid resuscitation and close observation continued during this period with good urine output and just one further episode of malaena. OGD was performed without complication and found no ulcers or bleeding points down to the fourth part of the duodenum. The patient's Hb recovered to $13.5 \mathrm{~g} / \mathrm{dL}$ and he became normotensive and euvolaemic. Anticoagulants were restarted and the patient was discharged four days after admission without further event with a GP follow-up scheduled.

\section{Conclusions}

This case report illustrates the need for prognostic scoring, close observation and aggressive fluid resuscitation in the management of GI bleeds.

\section{Abbreviations}

$\mathrm{AF}$, atrial fibrillation; $\mathrm{Hb}$, haemoglobin; INR, international normalised ratio; BMI, body mass index; AUGH, acute upper gastrointestinal haemorrhage; IV, intravenous.

\section{Consent}

Written informed consent was obtained from the patient for publication of this case report and accompanying images. A copy of the written consent is available for review by the Editor-in-Chief of this journal.

\section{Competing interests}

The authors declare that they have no competing interests.

\section{Author contributions}

Riaz Agha is the sole author, who saw the patient, assessed and treated him.
References

I. Rockall TA, Logan RF, Devlin HB, Northfield TC: Incidence of and mortality from acute upper gastrointestinal haemorrhage in the United Kingdom. Steering Committee and members of the National Audit of Acute Upper Gastrointestinal Haemorrhage. $\mathrm{Br}$ Med J 1995, 3 I I:222-226.

2. Agha R, Miller M, George ML, Sabharwal T: Arterial embolisation to control haemorrhage following colonoscopic polypectomy. International Journal of Surgery 2008, 6:420-42I.

3. Rockall TA, Logan RF, Devlin HB, Northfield TC: Selection of patients for early discharge or outpatient care after acute upper gastrointestinal haemorrhage. Lancet 1996, 347: I138-1140.

\section{Do you have a case to share?}

Submit your case report today

- Rapid peer review

- Fast publication

- PubMed indexing

- Inclusion in Cases Database

Any patient, any case, can teach us something

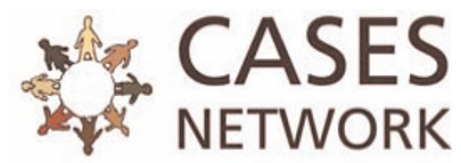

www.casesnetwork.com 